\title{
ROUGH MARCINKIEWICZ INTEGRAL OPERATORS
}

\author{
HUSSAIN AL-QASSEM and AHMAD AL-SALMAN
}

(Received 16 January 2001)

\begin{abstract}
We study the Marcinkiewicz integral operator $M_{\mathscr{P}} f(x)=\left(\int_{-\infty}^{\infty} \mid \int_{|y| \leq 2^{t}} f(x-\right.$ $\left.\mathscr{P}(y))\left.\left(\Omega(y) /|y|^{n-1}\right) d y\right|^{2} d t / 2^{2 t}\right)^{1 / 2}$, where $\mathscr{P}$ is a polynomial mapping from $\mathbb{R}^{n}$ into $\mathbb{R}^{d}$ and $\Omega$ is a homogeneous function of degree zero on $\mathbb{R}^{n}$ with mean value zero over the unit sphere $\mathbf{S}^{n-1}$. We prove an $L^{p}$ boundedness result of $M_{\mathscr{P}}$ for rough $\Omega$.
\end{abstract}

2000 Mathematics Subject Classification. 42B20, 42B15, 42B25.

1. Introduction. Let $\mathbb{R}^{n}, n \geq 2$ be the $n$-dimensional Euclidean space and $\mathbf{S}^{n-1}$ be the unit sphere in $\mathbb{R}^{n}$ equipped with the induced Lebesgue measure. Consider the Marcinkiewics integral operator

$$
\mu f(x)=\left(\int_{-\infty}^{\infty}\left|\mathbf{F}_{t}(x)\right|^{2} \frac{d t}{2^{2 t}}\right)^{1 / 2},
$$

where

$$
\mathbf{F}_{t}(x)=\int_{|x-y| \leq 2^{t}} f(y) \frac{\Omega(x-y)}{|x-y|^{n-1}} d y,
$$

and $\Omega$ is a homogeneous function of degree zero which has the following properties:

$$
\Omega \in L^{1}\left(\mathbf{S}^{n-1}\right), \quad \int_{\mathbf{S}^{n-1}} \Omega\left(y^{\prime}\right) d \sigma\left(y^{\prime}\right)=0 .
$$

When $\Omega \in \operatorname{Lip}_{\alpha}\left(\mathbf{S}^{n-1}\right),(0<\alpha \leq 1)$, Stein proved the $L^{p}$ boundedness of $\mu(f)$ for all $1<p \leq 2$. Subsequently, Benedek, Calderón, and Panzone proved the $L^{p}$ boundedness of $\mu(f)$ for all $1<p<\infty$ under the condition $\Omega \in C^{1}\left(\mathbf{S}^{n-1}\right)$ (see [2]).

The authors of [3] were able to prove the following result for the more general class of operators

$$
\mu_{P} f(x)=\left(\int_{-\infty}^{\infty}\left|\mathbf{F}_{P, t}(x)\right|^{2} \frac{d t}{2^{2 t}}\right)^{1 / 2}
$$

where

$$
\mathbf{F}_{P, t}(x)=\int_{|y| \leq 2^{t}} f\left(x-P(|y|) y^{\prime}\right) \frac{\Omega(y)}{|y|^{n-1}} d y
$$

and $P$ is a real-valued polynomial on $\mathbb{R}$ and satisfies $P(0)=0$.

THEOREM 1.1 (see [3]). Let $\alpha>0$, and $\Omega \in V_{\alpha}(n)$. Then the operator $\mu_{P}$ is bounded in $L^{p}\left(\mathbb{R}^{n}\right)$ for $(2 \alpha+2) /(2 \alpha+1)<p<2+2 \alpha$. 
In [1], Al-Salman and Pan studied the singular integral operator

$$
\mathbf{T}_{\Omega, \mathscr{P}} f(x)=\text { p.v. } \int_{\mathbb{R}^{n}} f(x-\mathscr{P}(y)) \frac{\Omega\left(y^{\prime}\right)}{|y|^{n}} d y,
$$

where $\mathscr{P}=\left(P_{1}, \ldots, P_{d}\right): \mathbb{R}^{n} \rightarrow \mathbb{R}^{d}$ is a polynomial mapping, $d \geq 1, n \geq 2$. The authors of [1] proved that $\mathbf{T}_{\Omega, \mathscr{P}}$ is bounded in $L^{p}\left(\mathbb{R}^{d}\right)$ whenever $(2+2 \alpha) /(1+2 \alpha)<p<2+2 \alpha$ and $\Omega \in W_{\alpha}(n)$. Here $W_{\alpha}(n)$ is a subspace of $L^{1}\left(\mathbf{S}^{n-1}\right)$ and its definition as well as the definition of $V_{\alpha}(n)$ will be reviewed in Section 2. It was shown in [1] that $W_{\alpha}(n)=$ $V_{\alpha}(n)$, if $n=2$ and it is a proper subspace of $V_{\alpha}(n)$ if $n \geq 3$.

Our purpose in this paper is to study the $L^{p}$ boundedness of the class of operators

$$
M_{\mathscr{P}} f(x)=\left(\int_{-\infty}^{\infty}\left|\mathbf{F}_{\mathscr{P}, t}(x)\right|^{2} \frac{d t}{2^{2 t}}\right)^{1 / 2},
$$

where

$$
\mathbf{F}_{\mathscr{P}, t}(x)=\int_{|y| \leq 2^{t}} f(x-\mathscr{P}(y)) \frac{\Omega(y)}{|y|^{n-1}} d y .
$$

Our main result in this paper is the following theorem.

THEOREM 1.2. Let $\alpha>0$, and $\Omega \in W_{\alpha}(n)$. Then the operator $M_{\odot}$ is bounded in $L^{p}\left(\mathbb{R}^{d}\right)$ for $(2 \alpha+2) /(2 \alpha+1)<p<2+2 \alpha$. The bound of $M_{\mathscr{P}} f$ is independent of the coefficients of $\left\{P_{j}\right\}$.

By [1, Theorem 3.1] and Theorem 1.2 we have the following corollary.

COROLlary 1.3. Let $\alpha>0, \Omega \in V_{\alpha}(2)$ and $\mathscr{P}: R^{2} \rightarrow R^{d}$. Then $M_{\mathscr{P}}$ is bounded in $L^{p}\left(R^{d}\right)$ for $(2 \alpha+2) /(2 \alpha+1)<p<2+2 \alpha$. The bound of $M_{\mathscr{P}}$ is independent of the coefficients of $\left\{P_{j}\right\}$.

2. Preparation. We start this section by recalling the following definition from [1].

DeFinition 2.1. For $\alpha>0, N \geq 1$, let $\tilde{\mathscr{V}}(n, N)=\bigcup_{m=1}^{N} \mathscr{V}(n, m)$ and let $W_{\alpha}(N, n)$ be the subspace of $L^{1}\left(\mathbf{S}^{n-1}\right)$ defined by

$$
W_{\alpha}(N, n)=\left\{\Omega \in L^{1}\left(\mathbf{S}^{n-1}\right): \int_{\mathbf{S}^{n-1}} \Omega\left(y^{\prime}\right) d \sigma\left(y^{\prime}\right)=0, M_{\alpha}(N, n)<\infty\right\},
$$

where

$$
\begin{aligned}
& M_{\alpha}(N, n) \\
& \quad=\max \left\{\int_{S^{n-1}}\left|\Omega\left(y^{\prime}\right)\right|\left(\log \frac{1}{\left|P\left(y^{\prime}\right)\right|}\right)^{1+\alpha} d \sigma\left(y^{\prime}\right): P \in \tilde{\mathscr{V}}(n, N) \text { with }\|P\|=1\right\} .
\end{aligned}
$$

For $\alpha>0$, we define $W_{\alpha}(n)$ to be

$$
W_{\alpha}(n)=\bigcap_{N=1}^{\infty} W_{\alpha}(N, n) .
$$

Also, for $\alpha>0$, we define $V_{\alpha}(n)$ by $V_{\alpha}(n)=W_{\alpha}(1, n)$ (see [6]). 
Here $\mathscr{V}(n, m)$ is the space of all real-valued homogeneous polynomials on $\mathbb{R}^{n}$ with degree equal to $m$ and with norm $\|\cdot\|$ defined by

$$
\left\|\sum_{|\alpha|=m} a_{\alpha} y^{\alpha}\right\|=\sum_{|\alpha|=m}\left|a_{\alpha}\right| .
$$

Now we need to recall the following results.

LEMMA 2.2 (see van der Corput [7]). Suppose $\phi$ and $\psi$ are real-valued and smooth in $(a, b)$, and that $\left|\phi^{(k)}(t)\right| \geq 1$ for all $t \in(a, b)$. Then the inequality

$$
\left|\int_{a}^{b} e^{-i \lambda \phi(t)} \psi(t) d t\right| \leq C_{k}|\lambda|^{-1 / k}\left[|\psi(b)|+\int_{a}^{b}\left|\psi^{\prime}(t)\right| d t\right],
$$

holds when

(i) $k \geq 2$, or

(ii) $k=1$ and $\phi^{\prime}$ is monotonic.

The bound $C_{k}$ is independent of $a, b, \phi$, and $\lambda$.

LEMMA 2.3 (see [7]). Let $\mathscr{P}=\left(P_{1}, \ldots, P_{d}\right)$ be a polynomial mapping from $\mathbb{R}^{n}$ into $\mathbb{R}^{d}$. Let $\operatorname{deg}(\mathscr{P})=\max _{1 \leq j \leq d} \operatorname{deg}\left(P_{j}\right)$. Suppose $\Omega \in L^{1}\left(\mathbf{S}^{n-1}\right)$ and

$$
\mu_{\Omega, \mathscr{P}} f(x)=\sup _{h>0}\left|\frac{1}{h^{n}} \int_{|y|<h} f(x-\mathscr{P}(y)) \Omega\left(y^{\prime}\right) d y\right| .
$$

Then for every $1<p \leq \infty$, there exists a constant $C_{p}>0$ which is independent of $\Omega$ and the coefficients of $\left\{P_{j}\right\}$ such that

$$
\left\|\mu_{\Omega, \Im} f\right\|_{p} \leq C_{p}\|\Omega\|_{L^{1}\left(\mathbf{S}^{n-1}\right)}\|f\|_{p}
$$

for every $f \in L^{p}\left(\mathbb{R}^{d}\right)$.

To each polynomial mapping $\mathscr{P}=\left(P_{1}, \ldots, P_{d}\right): \mathbb{R}^{n} \rightarrow \mathbb{R}^{d}$ with

$$
\operatorname{deg} \mathscr{P}=\max _{1 \leq j \leq d} \operatorname{deg} P_{j}=N, \quad d \geq 1, n \geq 2,
$$

we define a family of measures

$$
\left\{\vartheta_{t}^{l}, \lambda_{t}^{l}: l=0,1, \ldots, N, t \in \mathbb{R}\right\}
$$

as follows.

For $1 \leq j \leq d, 0 \leq l \leq N$ let $P_{j}=\sum_{|\alpha| \leq N} C_{j \alpha} y^{\alpha}$ and let $Q^{l}=\left(Q_{1}^{l}, \ldots, Q_{d}^{l}\right)$ where $Q_{j}^{l}=\sum_{|\alpha| \leq l} C_{j \alpha} y^{\alpha}$.

Now for $0 \leq l \leq N$ and $t \in \mathbb{R}$, let $\vartheta_{t}^{l}$ and $\lambda_{t}^{l}$ be the measures defined in the Fourier transform side by

$$
\begin{aligned}
& \left(\vartheta_{t}^{l} \hat{)}(\xi)=\int_{|y| \leq 2^{t}} e^{-2 \pi i \xi \cdot Q^{l}(y)} \frac{\Omega\left(y^{\prime}\right)}{|y|^{n-1}} \frac{d y}{2^{t}},\right. \\
& \left(\lambda_{t}^{l} \hat{)}(\xi)=\int_{|y| \leq 2^{t}} e^{-2 \pi i \xi \cdot Q^{l}(y)} \frac{\left|\Omega\left(y^{\prime}\right)\right|}{|y|^{n-1}} \frac{d y}{2^{t}} .\right.
\end{aligned}
$$


The maximal functions $\left(9^{l}\right) *$ defined by

$$
\left(g^{l}\right)^{*}(f)(x)=\sup _{t \in \mathbb{R}}\left|\lambda_{t}^{l} * f(x)\right|,
$$

for $l=0,1, \ldots, N$.

For later purposes, we need the following definition.

DeFinItion 2.4. For each $1 \leq l \leq N$, let $N_{l}=\left|\left\{\alpha \in \mathbf{N}^{n}:|\alpha|=l\right\}\right|$ and let $\{\alpha \in$ $\left.\mathbf{N}^{n}:|\alpha|=l\right\}=\left\{\alpha_{1}, \ldots, \alpha_{N_{l}}\right\}$. For each $1 \leq l \leq N$, define the linear transformations $L_{l}^{\alpha_{j}}: \mathbb{R}^{d} \rightarrow \mathbb{R}$ and $L_{l}: \mathbb{R}^{d} \rightarrow \mathbb{R}^{N_{l}}$ by

$$
\begin{aligned}
L_{l}^{\alpha_{j}}(\xi) & =\sum_{i=1}^{d}\left(C_{i, \alpha_{j} y^{\alpha_{j}}}\right) \xi_{i}, \quad j=1, \ldots, N_{l}, \\
L_{l}(\xi) & =\left(L_{l}^{\alpha_{1}}(\xi), \ldots, L_{l}^{\alpha_{N_{l}}}(\xi)\right) .
\end{aligned}
$$

To simplify the proof of our result we need the following lemma.

LEMMA 2.5. Let $\left\{\sigma_{t}^{l}: l=0,1, \ldots, N, t \in \mathbb{R}\right\}$ be a family of measures such that $\sigma_{t}^{0}=0$ for all $t \in \mathbb{R}$. Let $\mathbf{D}_{l}: \mathbb{R}^{n} \rightarrow \mathbb{R}^{d}, l=0,1, \ldots, N$ be linear transformations. Suppose that for all $t \in \mathbb{R}$ and $l=0,1, \ldots, N$, then

$$
\begin{gathered}
\left\|\sigma_{t}^{l}\right\| \leq C(l), \\
\left.\mid\left(\sigma_{t}^{l}\right) \hat{)} \xi\right) \mid \leq C \frac{M_{\alpha}}{\left(\log \left[c 2^{l t}\left|D_{l}(\xi)\right|\right]\right)^{1+\alpha}}, \\
\left.\mid\left(\sigma_{t}^{l}\right) \hat{(\xi)}-\left(\sigma_{t}^{l-1}\right) \hat{(} \xi\right)\left|\leq C 2^{l t}\right| D_{l}(\xi) \mid .
\end{gathered}
$$

Then there exists a family of measures $\left\{v_{t}^{l}: l=1, \ldots, N\right\}_{t \in \mathbb{R}}$ such that

$$
\begin{gathered}
\left\|v_{t}^{l}\right\| \leq C(l) \\
\mid\left(\nu_{t}^{l} \hat{)}(\xi) \mid \leq C \frac{M_{\alpha}}{\left(\log \left[c 2^{l t}\left|D_{l}(\xi)\right|\right]\right)^{1+\alpha}},\right. \\
\mid\left(\nu_{t}^{l} \hat{)}(\xi)\left|\leq C 2^{l t}\right| D_{l}(\xi) \mid,\right. \\
\sigma_{t}^{N}=\sum_{l=1}^{N} v_{t}^{l} .
\end{gathered}
$$

Proof. By [5, Lemma 6.1], for each $l=1, \ldots, N$ choose two nonsingular linear transformations

$$
A_{l}: \mathbb{R}^{r(l)} \longrightarrow \mathbb{R}^{d}, \quad B_{l}: \mathbb{R}^{d} \longrightarrow \mathbb{R}^{d}
$$

such that

$$
\left|A_{l} \pi_{r(l)}^{d} B_{l}(\xi)\right| \leq\left|D_{l}(\xi)\right| \leq N\left|A_{l} \pi_{r(l)}^{d} B_{l}(\xi)\right|, \quad \xi \in \mathbb{R}^{d}
$$

where $r(l)=\operatorname{rank}\left(D_{l}\right)$ and $\pi_{r(l)}^{d}$ is the projection operator from $\mathbb{R}^{d}$ into $\mathbb{R}^{r(l)}$. 
Now choose $\eta \in C_{0}^{\infty}(\mathbb{R})$ such that $\eta(t)=1$ for $|t| \leq 1 / 2$ and $\eta(t)=0$ for $|t| \geq 1$. Let $\varphi(t)=\phi\left(t^{2}\right)$ and let

$$
\begin{aligned}
\left(v_{t}^{l} \hat{)}(\xi)=\right. & \left(\sigma_{t}^{l}\right) \hat{(\xi)} \prod_{l<j \leq N} \varphi\left(\left|2^{t j} A_{j} \pi_{r(j)}^{d} B_{j}(\xi)\right|\right) \\
& -\left(\sigma_{t}^{l-1}\right) \hat{(\xi)} \prod_{l-1<j \leq N} \varphi\left(\left|2^{t j} A_{j} \pi_{r(j)}^{d} B_{j}(\xi)\right|\right)
\end{aligned}
$$

with the convention $\prod_{j \in \varnothing} a_{j}=1,1 \leq l \leq N$.

Hence, one can easily see that $\left\{\sigma_{t}^{l}: l=1, \ldots, N, t \in \mathbb{R}\right\}$ is the desired family of measures.

Now for the boundedness of the maximal functions $\left(9^{l}\right)^{*}, l=0,1, \ldots, N$, we have the following lemma whose proof is an easy consequence of Lemma 2.3, polar coordinates and Hölder's inequality:

LEMMA 2.6. For $l=1, \ldots, N$ and $p \in(1, \infty)$, there exists a constant $C_{p, l}$ which is independent of the coefficients of the polynomial components of the mapping $Q^{l}$ such that

$$
\left\|\left(9^{l}\right)^{*} f\right\|_{p} \leq C_{p, l}\|f\|_{p}
$$

3. Boundedness of some square functions. For a nonnegative $C^{\infty}$ radial function $\Phi$ on $\mathbb{R}^{n}$ with

$$
\operatorname{supp}(\Phi) \subset\left\{x \in \mathbb{R}^{n}: \frac{1}{2} \leq|x| \leq 2\right\}, \quad \int_{0}^{\infty} \frac{\Phi(t)}{t} d t=1,
$$

and for a linear transformation $\mathbf{L}: \mathbb{R}^{n} \rightarrow \mathbb{R}^{d}$, define the functions $\psi_{t}, t \in \mathbb{R}$ by $\hat{\psi}_{t}(y)=$ $\Phi\left(2{ }^{t} \mathbf{L}(y)\right)$.

For a family of measures $\left\{\sigma_{t}\right\}_{t \in \mathbb{R}}$, real number $u$ and $l \in \mathbb{N}$, let $\mathbf{J}_{u}^{l}(f)$ be the square function defined by

$$
\mathbf{J}_{u}^{l}(f)(x)=\left(\int_{-\infty}^{\infty}\left|\sigma_{t} * \psi_{l(t+u)} * f(x)\right|^{2} d t\right)^{1 / 2} .
$$

For such a square function we have the following theorem.

THEOREM 3.1. If $\left\{\sigma_{t}\right\}_{t \in \mathbb{R}}$ is a family of measures such that the corresponding maximal function

$$
\sigma^{*}(f)(x)=\sup _{t \in \mathbb{R}}|| \sigma_{t}|* f(x)|
$$

is bounded on $L^{p}\left(\mathbb{R}^{d}\right)$ for every $1<p<\infty$, then

$$
\left\|\mathbf{J}_{u}^{l}(f)\right\|_{L^{p}\left(\mathbb{R}^{d}\right)} \leq C_{p, l} \sqrt{\left\|\sigma^{*}\right\|\left\|_{(p / 2)^{\prime}} \sup _{t \in \mathbb{R}}\right\| \sigma_{t} \|}\|f\|_{L^{p}\left(\mathbb{R}^{d}\right)}
$$

for every $1<p<\infty$. Here $C_{p, l}$ is a constant that depends only on $p$ and the dimension of the underlying space. 
Proof. If $\sup _{t \in \mathbb{R}}\left\|\sigma_{t}\right\|=\infty$, then the inequality holds trivially. Thus we may assume that $\sup _{t \in \mathbb{R}}\left\|\sigma_{t}\right\|<\infty$. In this case we follow a similar argument as in [4]. Let $p>2$ and $q=(p / 2)^{\prime}$. Choose a nonnegative function $v \in L_{+}^{q}$ with $\|v\|_{q}=1$ such that

$$
\left\|\mathbf{J}_{u}^{l}(f)\right\|_{p}^{2}=\int_{\mathbb{R}^{d}}\left(\int_{-\infty}^{\infty}\left|\sigma_{t} * \psi_{l(t+u)} * f(x)\right|^{2} d t\right) v(x) d x .
$$

Thus it is easy to see that

$$
\begin{aligned}
\left\|\mathbf{J}_{u}^{l}(f)\right\|_{p}^{2} & \leq \sup _{t \in \mathbb{R}}\left\|\sigma_{t}\right\| \int_{-\infty}^{\infty} \int_{\mathbb{R}^{d}}\left|\psi_{l(t+u)} * f(z)\right|^{2} \sigma^{*}(v)(-z) d z d t \\
& \leq \sup _{t \in \mathbb{R}}\left\|\sigma_{t}\right\| \int_{\mathbb{R}^{d}}[g(f)]^{2}(z) \sigma^{*}(v)(-z) d z,
\end{aligned}
$$

where

$$
g(f)(x)=\left(\int_{-\infty}^{\infty}\left|\psi_{l(t+u)} * f(x)\right|^{2} d t\right)^{1 / 2} .
$$

Now since $\int_{\mathbb{R}^{d}} \psi_{t}(x) d x=0$, it is well known that

$$
\|g(f)\|_{p} \leq C_{p}\|f\|_{p} \quad \forall 1<p<\infty
$$

with constant $C_{p}$ that depends only on $p$ and the dimension of the underlying space.

Thus by (3.6) and Hölder's inequality we have

$$
\begin{aligned}
\left\|\mathbf{J}_{u}^{l}(f)\right\|_{p}^{2} & \leq \sup _{t \in \mathbb{R}}\left\|\sigma_{t}\right\|\|g(f)\|_{p}^{2}\left\|\sigma^{*}(u)\right\|_{q} \\
& \leq C_{p}^{2} \sup _{t \in \mathbb{R}}\left\|\sigma_{t}\right\|\left\|\sigma^{*}\right\|_{(p / 2)^{\prime}}\|f\|_{p}^{2} .
\end{aligned}
$$

Hence our result follows by taking the square root on both sides. The case $p<2$ follows by duality.

4. Proof of the main theorem. Let $\alpha>0, \Omega \in W_{\alpha}(n)$. Let $\mathscr{P}=\left(P_{1}, \ldots, P_{d}\right)$ be a polynomial mapping from $\mathbb{R}^{n}$ into $\mathbb{R}^{d}$ with $\operatorname{deg} \mathscr{P}=\max _{1 \leq j \leq d} \operatorname{deg} P_{j}=N$, where $d \geq 1$ and $n \geq 2$. For $0 \leq l \leq N$ let $N_{l}, Q^{l}, v_{t}^{l}, \lambda_{t}^{l}$, and $L_{l}$ be as in Section 3 .

The first step in our proof is to show that each $\vartheta_{t}^{l}, l=1, \ldots, N$ satisfies the hypotheses of Lemma 2.5, that is,

$$
\begin{gathered}
\left\|\vartheta_{t}^{l}\right\| \leq C(l), \\
\mid\left(\vartheta_{t}^{l} \hat{)}(\xi) \mid \leq C \frac{M_{\alpha}}{\left(\log \left[c 2^{l t}\left|L_{l}(\xi)\right|\right]\right)^{1+\alpha}},\right. \\
\mid\left(\vartheta_{t}^{l} \hat{)}(\xi)-\left(\vartheta_{t}^{l-1}\right)(\xi)\left|\leq C 2^{l t}\right| L_{l}(\xi) \mid .\right.
\end{gathered}
$$

One can easily see that (4.1) holds trivially. Using the cancellation property of $\Omega$, it is easy to see that (4.3) holds. Thus, we need only to verify (4.2). To see that, we notice that

$$
\mid\left(\vartheta_{t}^{l} \hat{)}(\xi)\left|\leq \int_{\mathbf{S}^{n-1}}\right| \Omega\left(y^{\prime}\right)|| \int_{0}^{1} e^{-2 \pi i \xi \cdot Q^{l}\left(2^{t} r y^{\prime}\right)} d r \mid d \sigma\left(y^{\prime}\right) .\right.
$$


Now the quantity $\xi \cdot Q^{l}\left(2^{t l} r y^{\prime}\right)$ can be written in the form

$$
\xi \cdot Q^{l}\left(2^{t l} r y^{\prime}\right)=2^{t l} r^{l} \lambda G^{l}\left(y^{\prime}\right)+\xi \cdot R\left(2^{t} r y^{\prime}\right),
$$

where $Q^{l}$ is a homogeneous polynomial of degree $l$ with $\left\|G^{l}\right\|=1, R$ is a polynomial of degree at most $l-1$ in the variable $r$,

$$
\lambda=\sum_{j=1}^{N_{l}}\left|L_{l}^{\alpha_{j}}(\xi)\right| \geq N_{l}\left|L_{l}(\xi)\right|
$$

and $\alpha_{1}, \ldots, \alpha_{N_{l}}$ are the constants that appeared in Section 2. Thus by van der Corput lemma, we have

$$
\left|\int_{0}^{1} e^{-2 \pi i \xi \cdot Q^{l}\left(2^{t} r y^{\prime}\right)} d r\right| \leq C \min \left\{1,\left(2^{t l}\left|L_{l}(\xi)\right|\left|G^{l}\left(y^{\prime}\right)\right|\right)^{-1 / l}\right\}
$$

and hence

$$
\left|\int_{0}^{1} e^{-2 \pi i \xi \cdot Q^{l}\left(2^{t} r y^{\prime}\right)} d r\right| \leq C \frac{\left[\log \left(c\left|G^{l}\left(y^{\prime}\right)\right|^{-1}\right)\right]^{1+\alpha}}{\left(\log \left[c 2^{t l}\left|L_{l}(\xi)\right|\right]\right)^{1+\alpha}}
$$

where $C$ is a constant independent of $t$ and $\xi$. Since $\Omega \in W_{\alpha}(n)$, the estimate (4.2) follows.

By Lemma 2.5, there exists a family of measures $\left\{v_{t}^{l}: l=1, \ldots, N, t \in \mathbb{R}\right\}$ such that

$$
\begin{gathered}
\left\|v_{t}^{l}\right\| \leq C(l) \\
\mid\left(v_{t}^{l} \hat{)}(\xi) \mid \leq C \frac{M_{\alpha}}{\left(\log \left[c 2^{l t}\left|L_{l}(\xi)\right|\right]\right)^{1+\alpha}}\right. \\
\mid\left(\nu_{t}^{l} \hat{)}(\xi)\left|\leq C 2^{l t}\right| L_{l}(\xi) \mid,\right. \\
\vartheta_{t}^{N}=\sum_{l=1}^{N} v_{t}^{l} .
\end{gathered}
$$

Also by Lemma 2.6 and the definition of $v_{t}^{l}$ (see the proof of Lemma 2.5), we have

$$
\left\|\left(v^{l}\right)^{*} f\right\|_{p} \leq C_{p, l}\|f\|_{p} \quad \forall 1<p<\infty .
$$

Now one can easily see that

$$
2^{-t} \mathbf{F}_{\mathscr{P}, t}(x)=\mathcal{\vartheta}_{t}^{N} * f(x)=\sum_{l=1}^{N} v_{t}^{l} * f(x) .
$$

Therefore,

$$
\left\|M_{\mathscr{P}} f\right\|_{p} \leq \sum_{l=1}^{N}\left\|M_{\mathscr{P}}^{l} f\right\|_{p}
$$

where

$$
M_{\mathscr{P}}^{l} f(x)=\left(\int_{-\infty}^{\infty}\left|v_{t}^{l} * f(x)\right|^{2} d t\right)^{1 / 2}
$$


Thus to show the boundedness of $M_{\mathscr{P}} f$, it suffices to show that

$$
\left\|M_{\mathscr{P}}^{l} f\right\|_{p} \leq C_{p, l}\|f\|_{p}
$$

for $p \in((2+2 \alpha) /(1+2 \alpha), 2+2 \alpha)$, and for all $l=1, \ldots, N$.

To show (4.17), we proceed as follows: let $\Phi$ and $\psi_{t}$ be as in Section 3 . Then

$$
\begin{aligned}
M_{\mathscr{P}}^{l} f(x) & =\log 2^{l}\left(\int_{-\infty}^{\infty}\left|\int_{-\infty}^{\infty} v_{t}^{l} * \psi_{l(t+u)} * f(x) d u\right|^{2} d t\right)^{1 / 2} \\
& \leq \log 2^{l} \int_{-\infty}^{\infty} S_{u}^{l} f(x) d u
\end{aligned}
$$

where

$$
S_{u}^{l} f(x)=\left(\int_{-\infty}^{\infty}\left|v_{t}^{l} * \psi_{l(t+u)} * f(x)\right|^{2} d t\right)^{1 / 2} .
$$

Now by (4.13) and Theorem 3.1, we have

$$
\left\|S_{u}^{l} f\right\|_{p} \leq C_{p}\|f\|_{p}
$$

for all $p \in(1, \infty)$ and for $l=1, \ldots, N$ which in turn implies that

$$
\int_{-1}^{1}\left\|S_{u}^{l} f\right\|_{p} d u \leq 2 C_{p}\|f\|_{p} \quad \forall p \in(1, \infty) .
$$

On the other hand, if $u \geq 1$, by the estimate (4.11) we have

$$
\begin{aligned}
\left\|S_{u}^{l} f\right\|_{2}^{2} & =\int_{\mathbb{R}^{d}} \int_{-\infty}^{\infty}\left|v_{t}^{l} * \psi_{l(t+u)} * f(x)\right|^{2} d t d x \\
& =\int_{-\infty}^{\infty} \int_{\mathbb{R}^{d}}\left(\Phi\left(2^{l t+l u} \mathbf{L}_{l}(\xi)\right)\right)^{2}\left|\left(v_{t}^{l}\right)(\xi)\right|^{2}|\hat{f}(\xi)|^{2} d \xi d t \\
& \leq 2^{2 l-2 l u} \int_{\mathbb{R}^{d}}|\hat{f}(\xi)|^{2}\left(\int_{\log \left(1 / 2^{l}\left|\mathbf{L}_{l}(\xi)\right|\right)-u}^{\log \left(2^{l} / \mathbf{L}_{l}(\xi) \mid\right)-u} d t\right) d \xi \\
& =2 \log 2^{l} 2^{2 l-2 l u}\|f\|_{2}^{2} .
\end{aligned}
$$

Thus

$$
\left\|S_{u}^{l} f\right\|_{2} \leq \sqrt{2 \log 2^{l}} 2^{l-l u}\|f\|_{2} .
$$

By interpolating between (4.20) and (4.23) we get

$$
\left\|S_{u}^{l} f\right\|_{p} \leq C_{p, l} 2^{\theta l-\theta l u}\|f\|_{p}
$$

for all $1<p<\infty$ and for some $\theta=\theta(p)>0$. Hence we have

$$
\int_{1}^{\infty}\left\|S_{u}^{l} f\right\|_{p} d u \leq C_{p}\|f\|_{p} \quad \text { for } p \in(1, \infty)
$$


Finally, if $u<-1$, by the estimate (4.10) and similar argument as in the case of $u \leq 1$, we get

$$
\left\|S_{u}^{l} f\right\|_{2} \leq C_{l}(|u|)^{-1-\alpha}\|f\|_{2}
$$

By interpolating between (4.26) and any $p \in(1, \infty)$ in (4.20), we get that, if $p \in$ $((2+2 \alpha) /(1+2 \alpha), 2+2 \alpha)$ there exists $\beta>0$ such that

$$
\left\|S_{u}^{l} f\right\|_{p} \leq C_{p}(|u|)^{-\beta}\|f\|_{p},
$$

which implies that

$$
\int_{-\infty}^{-1}\left\|S_{u}^{l} f\right\|_{p} d u \leq C_{p}\|f\|_{p}
$$

for $p \in((2+2 \alpha) /(1+2 \alpha), 2+2 \alpha)$.

Hence by combining (4.18), (4.21), (4.25), and (4.28) we get (4.17).

ACKNOWLEDGEMENT. The publication of this paper was supported by Yarmouk University Research Council.

\section{REFERENCES}

[1] A. Al-Salman and Y. Pan, Singular integrals with rough kernels, to appear in Canad. Math. Bull.

[2] A. Benedek, A. P. Calderón, and R. Panzone, Convolution operators on Banach space valued functions, Proc. Nat. Acad. Sci. U.S.A. 48 (1962), 356-365. MR 24\#A3479. Zbl 103.33402.

[3] J. Chen, D. Fan, and Y. Pan, A note on a Marcinkiewics integral operator, to appear in Math. Nachr.

[4] J. Duoandikoetxea and J. L. Rubio de Francia, Maximal and singular integral operators via Fourier transform estimates, Invent. Math. 84 (1986), no. 3, 541-561. MR 87f:42046. Zbl 568.42012.

[5] D. Fan and Y. Pan, Singular integral operators with rough kernels supported by subvarieties, Amer. J. Math. 119 (1997), no. 4, 799-839. MR 99c:42029. Zbl 0899.42002.

[6] L. Grafakos and A. Stefanov, $L^{p}$ bounds for singular integrals and maximal singular integrals with rough kernels, Indiana Univ. Math. J. 47 (1998), no. 2, 455-469. MR 99i:42019. Zbl 913.42014.

[7] E. M. Stein, Harmonic Analysis: Real-Variable Methods, Orthogonality, and Oscillatory Integrals, Princeton Mathematical Series, vol. 43, Princeton University Press, New Jersey, 1993. MR 95c:42002. Zbl 821.42001.

HuSSAIN AL-QASSEM: DEPARTMENT OF MATHEMATICS, YARMOUK UNIVERSITY, IRBID, JORDAN E-mail address: husseink@yu. edu. jo

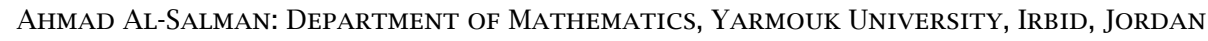
E-mail address: a1sa7man@yu. edu . jo 


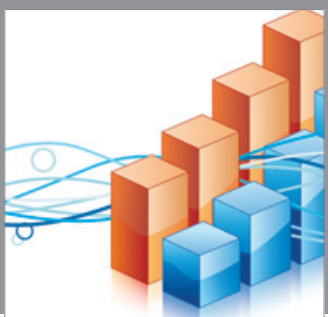

Advances in

Operations Research

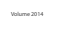

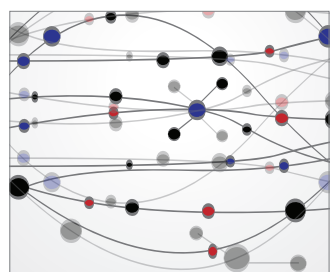

\section{The Scientific} World Journal
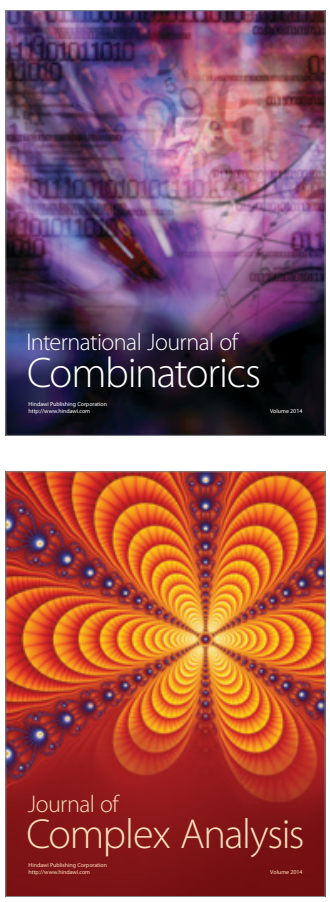

International Journal of

Mathematics and

Mathematical

Sciences
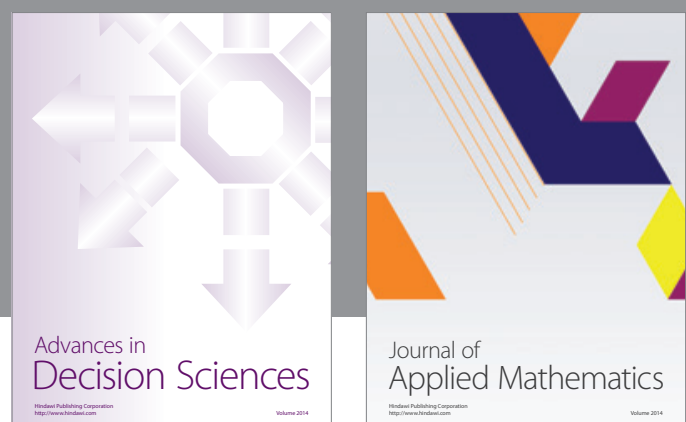

Journal of

Applied Mathematics
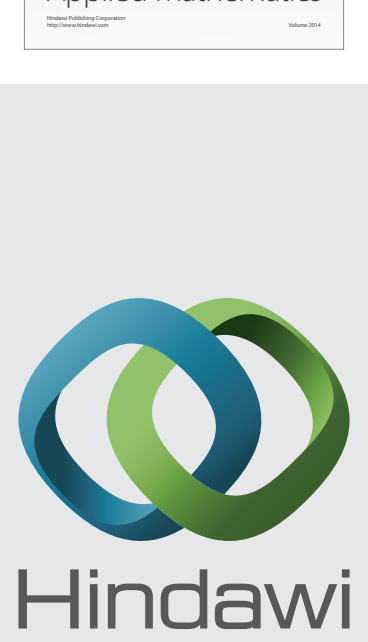

Submit your manuscripts at http://www.hindawi.com
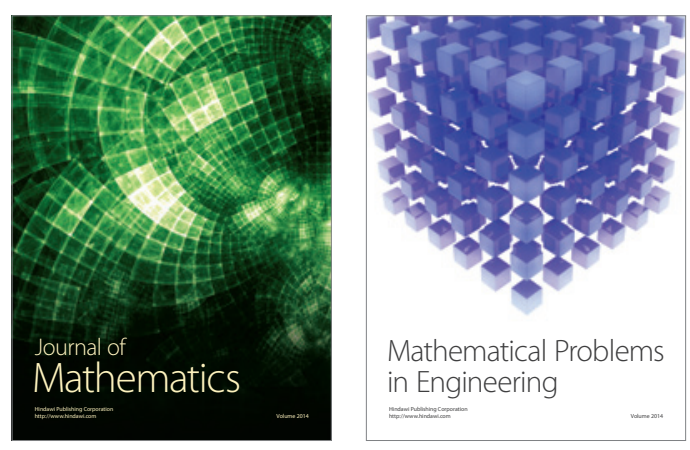

Mathematical Problems in Engineering
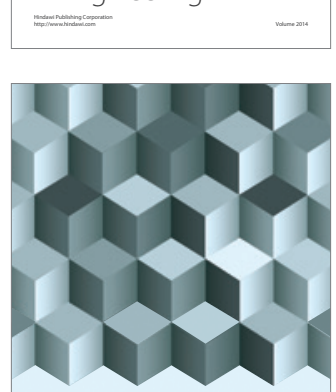

Journal of

Function Spaces
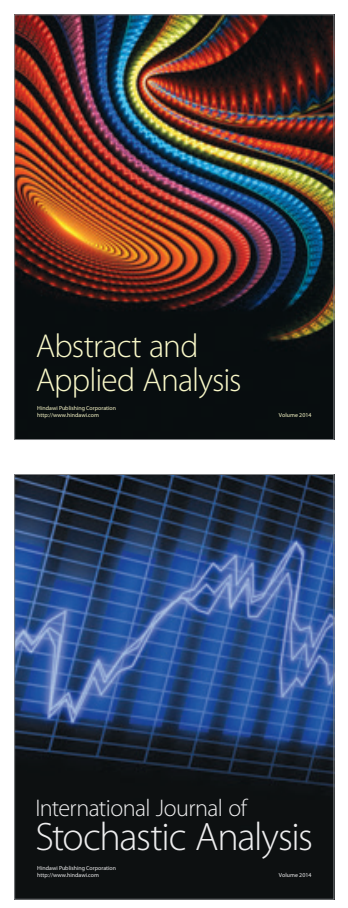

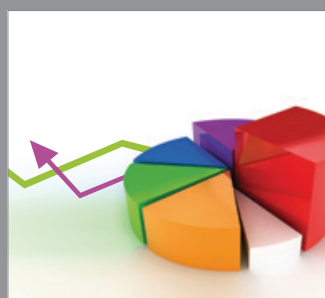

ournal of

Probability and Statistics

Promensencen
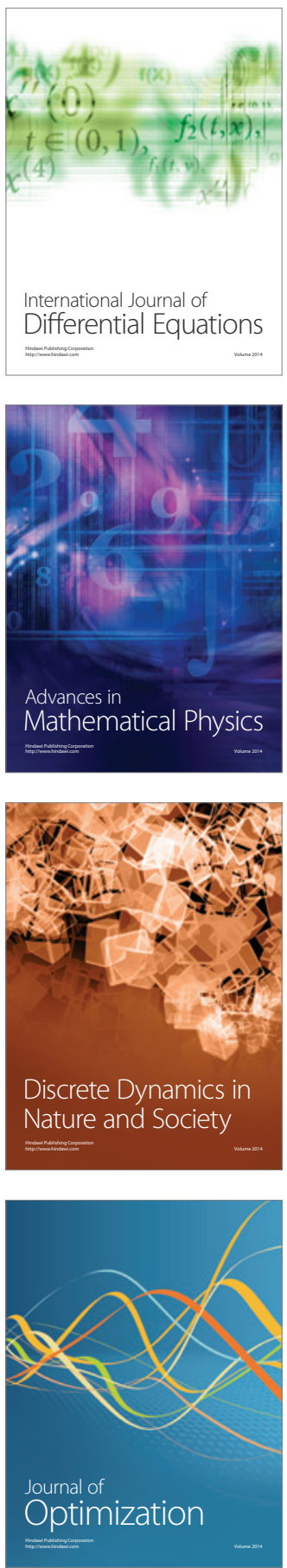